\title{
Resonance Raman Spectra of Erythrocytes: Vibron Model
}

\author{
J. VIJAYASEKHAR \\ Department of Mathematics, School of Technology, GITAM, Hyderabad, India \\ ${ }^{*}$ Corresponding author E-mail: vijayjaliparthi@gmail.com \\ http://dx.doi.org/10.13005/ojc/340561
}

(Received: January 03, 2018; Accepted: September 01, 2018)

\begin{abstract}
In this paper, vibrational spectra (infrared and Raman) of oxygenated and deoxynated functional erythrocytes are calculated using theoretical method "Vibron model" in the one-dimensional $[U(2)]$ framework. The determined vibrational modes by Vibron model are compared with experimental data. It has been observed that results from the theoretical model reveal near to the exact, reliable with the experimental data.
\end{abstract}

Keywords: Lie algebras, Resonance Raman spectra, Vibron model, Erythrocytes.

\section{INTRODUCTION}

Molecular spectroscopy is a key tool for the explanation of molecular structure. The study of fundamental and higher overtones of molecule helps in better understanding of the molecular structure, which might have a great importance in medical science. This spectra is useful to analyse biological molecules for identifying diseases in near the beginning stage and also useful to compare in vivo experimental results. The frame of new experimental methods to identify higher vibrational excitations in polyatomic molecules requires theoretical models (example, Vibron model) for their interpretation. Vibrational spectra of molecules are analysed with the help of two well-known approaches Dunham expansion and Potential approach. The most serious drawback of these approaches is that large number of parameters required for polyatomic molecules.
To overcome this difficulty, we consider the Vibron mode $^{1,2}$. In 1991, lachello presented Vibron model in the study of spectra of molecules ${ }^{3,4,5,6}$.

\section{Vibron model}

The Vibron model (Hamiltonian) for polyatomic molecule is

$H=E_{0}+\sum_{\mathrm{i}=1}^{\mathrm{n}} A_{\mathrm{i}} C_{\mathrm{i}}+\sum_{\mathrm{i}<\mathrm{j}}^{\mathrm{n}} A_{\mathrm{j}} C_{\mathrm{ij}}+\sum_{\mathrm{i}<\mathrm{j}}^{\mathrm{n}} \lambda_{\mathrm{ij}} M_{\mathrm{ij}}$.

Here $\left(A_{i}, A_{i j}, A_{i j}\right)$ are parameters (algebraic) and $\left(C_{i}, C_{i j}, M_{i j}\right)$ are algebraic operators, which are differ from molecule to molecule. Algebraic operators, $C_{i}$ and $C_{i j}$ are invariant operators associated with uncoupled and coupled bonds with diagonal matrix elements. The Majorana operator, $\mathrm{M}_{\mathrm{ij}}$ associated with coupling schemes. Algebraic parameters can be determined by spectroscopic data. Algebraic operators can be determined by 
$\left\langle C_{\mathrm{i}}\right\rangle=-4\left(N_{\mathrm{i}} \mathrm{v}_{\mathrm{i}}-\mathrm{v}_{\mathrm{i}}^{2}\right)$

$\left\langle N_{\mathrm{i}}, \mathrm{v}_{\mathrm{i}} ; N_{\mathrm{j}}, \mathrm{v}_{\mathrm{j}}\left|C_{\mathrm{ij}}\right| N_{\mathrm{i}}, \mathrm{v}_{\mathrm{i}} ; N_{\mathrm{j}}, \mathrm{v}_{\mathrm{j}}\right\rangle=4\left[\left(\mathrm{v}_{\mathrm{i}}+\mathrm{v}_{\mathrm{j}}\right)^{2}-\left(\mathrm{v}_{\mathrm{i}}+\mathrm{v}_{\mathrm{j}}\right)\left(N_{\mathrm{i}}+N_{\mathrm{j}}\right)\right]$

$\left\langle N_{\mathrm{i}}, \mathrm{v}_{\mathrm{i}} ; N_{\mathrm{j}}, \mathrm{v}_{\mathrm{j}}\left|M_{\mathrm{ij}}\right| N_{\mathrm{i}}, \mathrm{v}_{\mathrm{i}} ; N_{\mathrm{j}}, \mathrm{v}_{\mathrm{j}}\right\rangle=\left(N_{\mathrm{i}} \mathrm{v}_{\mathrm{j}}+N_{\mathrm{j}} \mathrm{v}_{\mathrm{i}}-2 \mathrm{v}_{\mathrm{i}} \mathrm{v}_{\mathrm{j}}\right)$

$\left\langle N_{\mathrm{i}}, \mathrm{v}_{\mathrm{i}}+1 ; N_{\mathrm{j}} \mathrm{v}_{\mathrm{j}}-1\left|M_{\mathrm{ij}}\right| N_{\mathrm{i}}, \mathrm{v}_{\mathrm{i}} ; N_{\mathrm{j}} \mathrm{v}_{\mathrm{j}}\right\rangle=-\left[\mathrm{v}_{\mathrm{j}}\left(\mathrm{v}_{\mathrm{i}}+1\right)\left(N_{\mathrm{i}}-\mathrm{v}_{\mathrm{i}}\right)\left(N_{\mathrm{j}}-\mathrm{v}_{\mathrm{j}}+1\right)\right]^{1 / 2}$

$\left\langle N_{\mathrm{i}}, \mathrm{v}_{\mathrm{i}}-\mathrm{l} ; N_{\mathrm{j}}, \mathrm{v}_{\mathrm{j}}+1\left|M_{\mathrm{ij}}\right| N_{\mathrm{i}}, \mathrm{v}_{\mathrm{i}} ; N_{\mathrm{j}}, \mathrm{v}_{\mathrm{j}}\right\rangle=-\left[\mathrm{v}_{\mathrm{i}}\left(\mathrm{v}_{\mathrm{j}}+1\right)\left(N_{\mathrm{j}}-\mathrm{v}_{\mathrm{j}}\right)\left(N_{\mathrm{i}}-\mathrm{v}_{\mathrm{i}}+1\right)\right]^{1 / 2}$.

In above equations, $N_{\mathrm{i}}, \mathrm{v}_{\mathrm{i}}(\mathrm{i}=1,2, \ldots)$ represents Vibron and vibrational quantum numbers respectively.

The vibron number $N_{\mathrm{i}}$ for stretching bonds of molecule will be calculated by

$N_{\mathrm{i}}=\frac{\omega_{e}}{\omega_{e} x_{e}}-1, \mathrm{i}=1,2,3, \ldots \quad\left(N_{\mathrm{i}}=N_{\mathrm{j}}\right)$
Here $\omega_{\mathrm{e}}$ and $\omega_{\mathrm{e}} \chi_{\mathrm{e}}$ are the spectroscopic constants. The initial guess value for the parameter $A_{i}$ is obtained by using the energy equation, which is known as,

$E(\mathrm{v}=1)=-4 A_{\mathrm{i}}\left(N_{\mathrm{i}}-1\right)$

The first guess for $A_{i j}$ can be taken as zero. The parameter obtained from the relation,

$\lambda_{\mathrm{ij}}=\frac{\left|E_{\mathrm{i}}-E_{\mathrm{j}}\right|}{k N}(k=2,6)$

\section{RESULTS}

From the group theory point of view, the molecule of oxygenated and deoxygenated erythrocytes is a square planar structure with the $D_{4 h}$ symmetry point group ${ }^{8}$. We considered a experimental data from high-quality Raman speectra of red blood cells in both the oxygenated and the deoxygenated states for cell at $782 \mathrm{~nm}^{7}$.

Table 1: Vibrational spectra of erythrocytes

\begin{tabular}{|c|c|c|c|}
\hline \multirow[t]{2}{*}{ Symmetry Species ${ }^{3}$} & \multirow[t]{2}{*}{ Vibrational mode } & \multicolumn{2}{|c|}{ Vibrational frequencies $\left(\mathrm{cm}^{-1}\right)$} \\
\hline & & Calculated & Experimental $^{7}$ \\
\hline & oxynated erythrocytes & & \\
\hline$B_{1 g}$ & $\left(\mathrm{C}_{\mathrm{m}}-\mathrm{H}\right)$ & 1305.2346 & 1306 \\
\hline$E_{u}$ & $\begin{array}{c}\left(\mathrm{C}_{\mathrm{m}}-\mathrm{H}\right) \\
\text { deoxynated erythrocytes }\end{array}$ & 1250.0429 & 1248 \\
\hline$B_{1 g}$ & $\left(\mathrm{C}_{\mathrm{m}}-\mathrm{H}\right)$ & 1303.0086 & 1305 \\
\hline$E_{u}$ & $\left(\mathrm{C}_{\mathrm{m}}-\mathrm{H}\right)$ & 1244.9821 & 1248 \\
\hline
\end{tabular}

Table 2: Algebraic parameters

\begin{tabular}{ccc}
\hline Algebraic parameters & oxynated & deoxynated \\
\hline$A$ & -9.3485 & -10.5453 \\
$\lambda$ & 0.3581 & 0.1277 \\
$N$ & 44 & 44
\end{tabular}

Values of algebraic parameters in $\mathrm{cm}^{-1}$, but $N$ is dimensionless

\section{CONCLUSION}

In this paper we have calculated the vibrational frequencies of oxynated and deoxynated erythrocytes for the vibrational mode $(\mathrm{Cm}-\mathrm{H})$ by Vibron model and also compared with experimental data. These calculations shows that this model is an alternative appoarch of other theoretical models like Ab initio methods.

\section{REFERENCES}

1. lachello, F.; Levine, R. D. Oxford University Press, Oxford., 1995.

2. Oss, S. Adv. Chem. Phys., 1996, 93, 455-649.

3. Karumuri, S. R.; Vijayasekhar, J.; Sreeram, V.; Uma Maheswara Rao, V.; Basaveswara Rao, M. V. J. Mol. Spectrosc., 2011, 269, 119-123.

4. Karumuri, S. R.; Srinivas, G.; Vijayasekhar, J.; Rao, V. U. M.; Srinivas, Y.; Sunil Babu, K.; Kumar, V. S. S.; Hanumaiah, A. Chin. Phy. B.,
2013, 22(9), 090304(1-8).

5. Vijayasekhar, J.; Karumuri, S. R.; Rao, V. U. M. Natural Science., 2012, 4(10), 792-796.

6. Karumuri, S. R.; Rao, V. U. M.; Vijayasekhar, J. Proc. Materials Science., 2015, 10, 737-747.

7. Bayden R. Wood; Peter Caspers; Gerwin J. Pupples. Anal. Bioanal. Chem., 2007, 387, 1691-1703.

8. Karumuri, S. R.; Sravani, K.G. Mol. Phys., 2016, 114(5), 643-649. 